\title{
IMAGE INFORMATICS STRATEGIES FOR DECIPHERING NEURONAL NETWORK CONNECTIVITY
}

\author{
Jan R. Detrez ${ }^{1}$, Peter Verstraelen ${ }^{1}$, Titia Gebuis ${ }^{2}$, Marlies Verschuuren ${ }^{1}$, Jacobine Kuijlaars ${ }^{3}$, Xavier Langlois ${ }^{3}$, \\ Rony Nuydens ${ }^{3}$, Jean-Pierre Timmermans ${ }^{1}$, Winnok H. De Vos ${ }^{1,4}$ \\ ${ }^{1}$ Laboratory of Cell Biology and Histology, Dept. Veterinary Sciences, University of Antwerp, Antwerp, \\ Belgium \\ ${ }^{2}$ Department of Molecular and Cellular Neurobiology, Center for Neurogenomics and Cognitive Research, VU \\ University Amsterdam, Amsterdam, The Netherlands \\ ${ }^{3}$ Neuroscience Dept., Janssen Research and Development, Beerse, Belgium \\ ${ }^{4}$ Cell Systems and Imaging, Dept. Molecular Biotechnology, University of Ghent, Belgium
}

\begin{abstract}
Brain function relies on an intricate network of highly dynamic neuronal connections that rewires dramatically under the impulse of various external cues and pathological conditions. Among the neuronal structures that show morphological plasticity are neurites, synapses, dendritic spines and even nuclei. This structural remodelling is directly connected with functional changes such as intercellular communication and the associated calcium-bursting behaviour. In vitro cultured neuronal networks are valuable models for studying these morpho-functional changes. Owing to the automation and standardisation of both image acquisition and image analysis, it has become possible to extract statistically relevant readout from such networks. Here, we focus on the current state-of-the-art in image informatics that enables quantitative microscopic interrogation of neuronal networks. We describe the major correlates of neuronal connectivity and present workflows for analysing them. Finally, we provide an outlook on the challenges that remain to be addressed, and discuss how imaging algorithms can be extended beyond in vitro imaging studies.
\end{abstract}




\section{INTRODUCTION}

Development of the central nervous system entails formation and maintenance of intricate neuronal networks. Synaptic activity and the associated opening of gated ion channels initiate precisely calibrated calcium transients in neuronal cells, which drive short-term and longterm morphological changes, such as dendritic growth and arborisation ${ }^{1}$. This dynamic, cytoskeleton-based remodelling of neuronal appendages, also known as neuronal plasticity, is a key process for virtually all long-lasting adaptations of the brain, such as learning, addiction or chronic pain sensation ${ }^{2}$. While resulting from very different molecular triggers (e.g. the production of toxic protein oligomers, cytoskeletal dysregulation, etc.), disrupted neuronal plasticity represents a pathological hallmark that is shared by numerous psychiatric and neurodegenerative diseases, including schizophrenia, autism spectrum disorder and Alzheimer's disease $^{3,4}$. Thus, understanding the intricacies of neuronal connectivity may not only be instrumental in gaining insights into its physiological importance, but also in resolving stages of disease development.

\subsection{Models for studying neuronal connectivity}

Because of the complexity and long-distance wiring of neurons in the brain, neuronal connectivity is ideally studied within the entire organ. Boosted by the differential power of stochastic multispectral labelling technologies like Brainbow and derivatives ${ }^{5}$, multiple imaging approaches have been developed that enable connectivity studies in whole fixed and even living brain. Microscopic imaging in awake animals has been achieved with implanted cranial windows that can be accessed after restraining the animal, or using miniature head-mounted microscopes in freely moving animals ${ }^{6,7}$. However, the imaging depth of such studies is limited to the optical penetration power of multi-photon microscopes $(\sim 1 \mathrm{~mm})^{8}$. Recent advances in tissue clearing and re-invention of light-sheet illumination microscopy have enabled 3D microscopic imaging of intact fixed brains at unprecedented speed ${ }^{9}$. One of the aims of these efforts is to build a digital atlas from the vast datasets to enable mapping the connectivity between and within brain regions ${ }^{10,11}$. However, the methods for acquiring and analysing such datasets are far from standard, the size of the datasets is massive and interpretation, let alone quantification, is non-trivial ${ }^{12}$.

For live cell imaging studies, acute or organotypic brain slices circumvent the need for extended animal suffering and monitoring of multiple physiological parameters typically accompanying in vivo manipulation ${ }^{13}$. While maintaining a reasonable level of tissue architecture, this approach improves the experimental access and allows precise control of the extracellular environment. Nevertheless, afferent signals from distant brain regions are inevitably lost and physiological processes cannot be associated with behavioural information. A major disadvantage that is shared by both intact brain and slice model approaches is that it is difficult to standardize the quantitative readout when it comes down to studying connectivity. The inter-individual variability between model organisms creates a tremendous bias and impedes easy extraction of morphological and functional cues. This, together with the need for large amounts of biological material, precludes their use from routine screening in preclinical drug screening campaigns, which is why in vitro models have been established. The advantage of using neuronal cells is that multiple cell cultures can be grown in parallel, allowing multiplex experiments with internal controls. Although existing 3D anatomical connections are lost during the preparation of primary neurons (e.g. extracted from mouse embryos), 
the cells preserve numerous morphological and functional properties of in vivo neuronal networks $^{14-17}$. For example, it has been shown that primary cultures recapitulate synchronous calcium bursting behaviour, when cultured in a 96-well plate format, making this platform highly attractive for high-throughput pharmacological and genetic manipulation ${ }^{18,19}$. To overcome species differences, recent efforts have also led to the use of human iPSC-derived neuronal cultures ${ }^{20,21}$. iPSC technology circumvents ethical obstructions regarding human embryonic stem cells and allows cultivating patient-derived neurons, thereby eliminating the need for artificial disease models.

\subsection{Correlates of neuronal connectivity}

Cultivated neuronal networks display both morphological and functional features that can be used to quantitatively describe the degree of connectivity (Fig. 1). The outgrowth of axons and dendrites, collectively called neurites, is a morphological feature that provides information about the general health of the neurons and the connectivity within the neuronal network. Consequently, this feature has been used in high-throughput compound toxicity screening and safety evaluation of drugs and environmental chemicals ${ }^{22-24}$. Different approaches to quantify neuronal morphology (e.g. neurite outgrowth, neurite bifurcations, Sholl analysis) are discussed in paragraph 2.2.

Neuronal communication is established through the formation of synapses. A synapse consists of three major compartments: a presynaptic compartment, a postsynaptic compartment and the synaptic cleft. Pre- and postsynaptic compartments are highly specialized morphological structures containing specific proteins that can be used as markers for assessing neuronal connectivity. As such, fluorescent labelling and quantification of synaptic proteins may provide valuable information about the number? of synapses, and therefore serve as an indicator of the connectivity in the network. This is discussed in paragraph 2.3.

While inhibitory synapses are made directly on the dendritic shaft, the postsynaptic compartment of excitatory synapses is predominantly located on highly specialized structures, called dendritic spines. These spines are small $(0.5-3 \mu \mathrm{m})$ protrusions from the dendritic shaft that were first described by Ramon y Cajal in $1891^{25}$. The exact functions of spines are still debated, but the general view is that they compartmentalize the local electrical and biochemical processes of a single synapse ${ }^{26}$. They are highly dynamic structures that change in shape, volume and density in response to cues that influence synaptic strength. Throughout the continuum of spine shapes, different morphological stages (thin, stubby or mushroom shape) can be discriminated, which can change within a matter of minutes via rearrangements of the actin cytoskeleton (Fig. 1) ${ }^{27-29}$. The synaptic receptors on spines are connected to a local cytoskeletal network via the assembly of scaffold proteins, called the postsynaptic density (PSD). Thin spines contain relatively small PSDs and emerge and disappear over a few days, whereas mushroom spines, with larger PSDs may persist for months. Spine density and morphology are becoming increasingly popular as readouts for neuronal network connectivity and alterations in both features have been described in numerous neurological disorders, including Alzheimer's disease, schizophrenia, intellectual disabilities and autism spectrum disorders ${ }^{4}$.

While morphological correlates provide a static impression of connectivity, they do not inform on the actual synaptic communication taking place within a network. It is only by direct assessment of this electrical activity that one can grasp the true degree of functional connectivity (discussed in paragraph 3). Cultivated neurons are known to exhibit spontaneous electrical activity, which tends to evolve from stochastic activity of individual neurons into ro- 
bust, synchronized network activity ${ }^{14,18}$. Neuronal electrical activity can be visualized by means of voltage or calcium sensors, both of which are available as synthetic dyes or genetically encoded fluorescent proteins ${ }^{30-33}$. Such a functional approach not only allows assessing the effect of chronic treatments on neuronal connectivity, but can also provide information about acute responses to pharmacological perturbations.
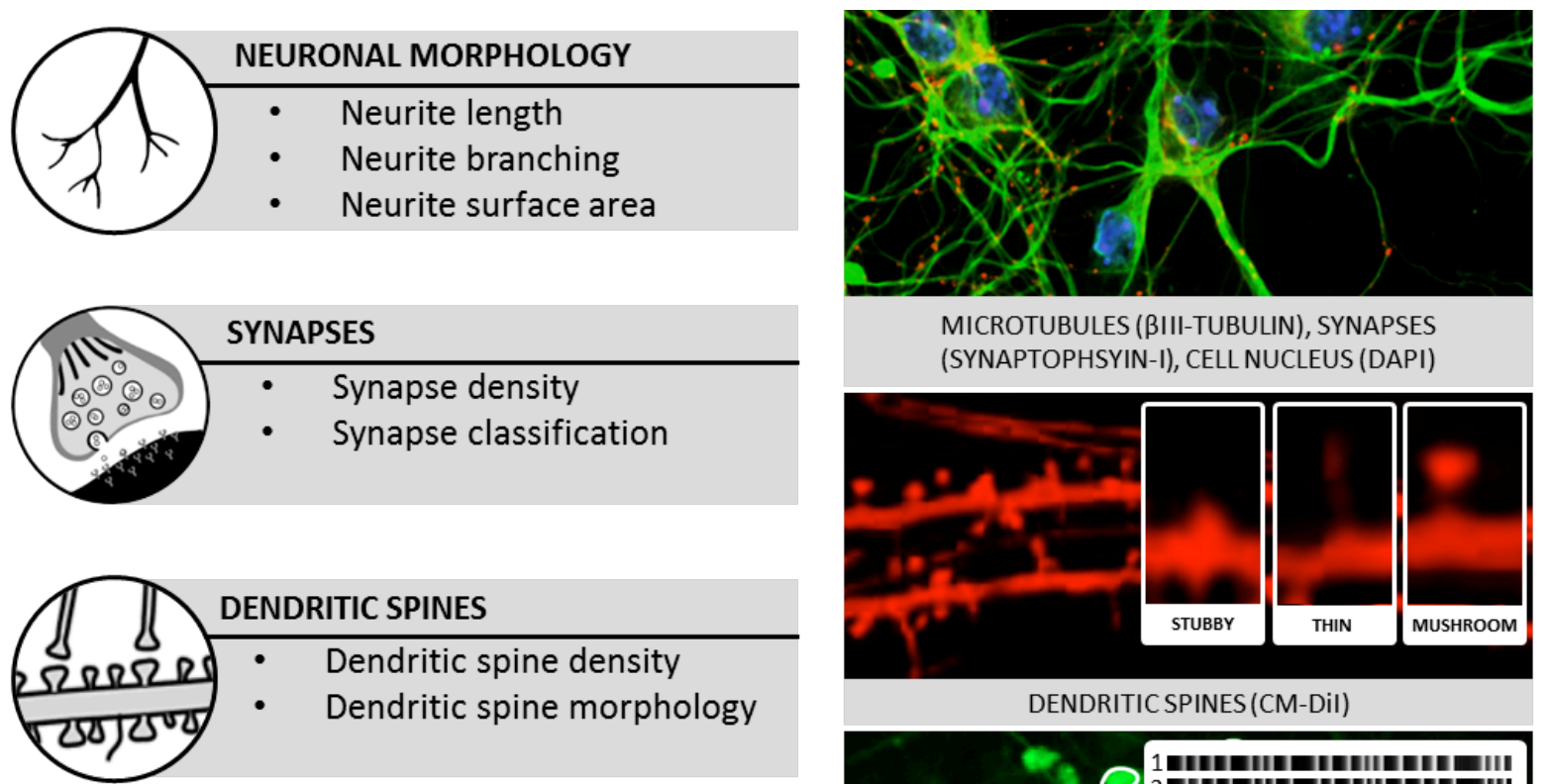

MICROTUBULES ( $\beta$ III-TUBULIN), SYNAPSES (SYNAPTOPHSYIN-I), CELL NUCLEUS (DAPI)

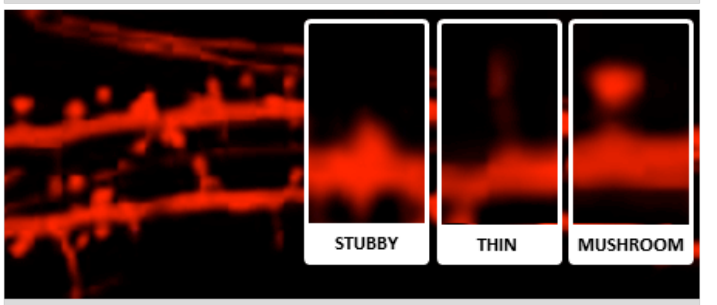

DENDRITIC SPINES(CM-Dil)

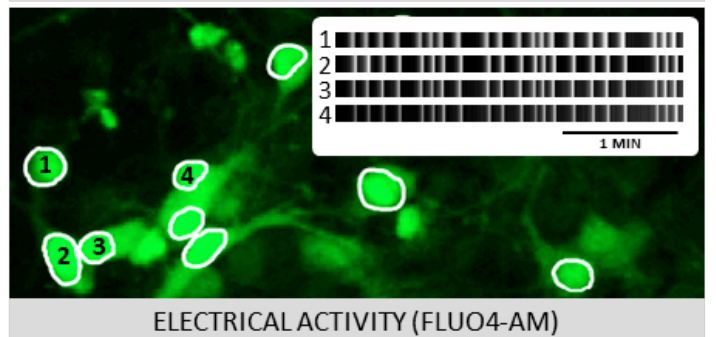

Figure 1. Correlates of neuronal network connectivity.

The main morphological (neuronal morphology, synapses and dendritic spines) and functional (calcium activity) correlates of in vitro neuronal network connectivity are depicted. Immunocytochemical labelling of cytoskeletal proteins, such as $\beta$-III-tubulin, allows quantifying the neuronal morphology, while labelling of synaptic proteins provides information about the synapse density or the type of neurotransmitter they process. Dendritic spines are specialized compartments that contain excitatory synapses and can be highlighted with lipophilic dyes (e.g. CM-DiI). Both density and morphology of spines correlate with synaptic strength and hence network connectivity. Calcium imaging (e.g. using the calcium-sensitive dye Fluo4-AM) allows studying the spontaneous electrical activity of neurons.

\subsection{From snapshots to numbers: towards high-content neuro-imaging}

Both primary and iPSC-derived neuronal networks can be cultivated in multi-well plates, starting from a limited amount of biological material. In combination with automated fluorescence microscopy, these networks make an attractive model for upscaling to a high-content screening (HCS) platform ${ }^{19,34}$. Of vital importance for such a platform is robust measurement of the endpoint of interest. Manual quantification is not only labour-intensive, but also prone to observer bias, which hampers reproducibility of the data. To eliminate this bias and boost throughput, automation of image analysis is inevitable. However, the design and implementation of generic automated image analyses are non-trivial since the experimental conditions, 
such as microscope settings, type of stains, cell type and cell densities that are used, introduce a strong variability in image quality ${ }^{35}$. Nevertheless, with sufficient standardization of the sample preparation and image acquisition protocols, and adequate pre-processing of the raw image datasets, the major correlates of neuronal connectivity can be quantified in an unbiased way. In the following paragraphs, we discuss the main image analysis strategies for quantification of morphological and functional endpoints.

\section{MEASURING MORPHOLOGICAL CORRELATES: FROM NETWORKS TO SPINES}

As mentioned above, neurons exhibit strong morphological plasticity. Relevant dynamic changes that can be quantified are neuronal morphology, synapse development and the emergence and remodelling of dendritic spines. The analysis of each of these features differs, but they all rely on a generic workflow that consists of four major steps, namely pre-processing (image restoration), segmentation (object detection), rectification (visual verification and correction) and analysis (feature extraction). We will first briefly introduce some of the generic methods in image pre-processing that apply to all analysis pipelines, after which we will focus on the more dedicated algorithms for extracting morphological data.

\subsection{Basic image pre-processing}

The principal task of image pre-processing is to correct for systematic errors and imperfections that have been introduced by the image acquisition system. These errors include image blur (imposed by the point-spread function), noise (photon and detector noise) and intensity gradients (due to spatiotemporal illumination inhomogeneity). Various algorithms have been introduced to tackle these issues. One of the first pre-processing steps that is often used is deconvolution, ${ }^{36}$ which is also known as image restoration since it aims at reversing the image formation process, thereby improving the signal-to-noise ratio (SNR) and image resolution ${ }^{37}$. Image noise predominantly results from the stochastic nature of the photon-counting process at the detectors (i.e. Poisson noise), and the intrinsic thermal and electronic fluctuations of the acquisition devices (i.e. Gaussian noise). Gaussian noise can be easily removed by conventional spatial filtering techniques (e.g. mean filtering or Gaussian smoothing). This works fast, but generally tends to reduce noise at the expense of sharpness. More advanced (e.g. wavelet-based $^{38}$ ) methods that correct for Poisson noise have been described as well. Heterogeneous illumination and nonlinearities in the acquisition path are usually corrected for by subtracting an image of an empty region (flat-field correction) or by local background subtraction (pseudo-flat field correction).

\subsection{Neuronal morphology}

The necessity for analysing neuronal morphology has led to the development of a variety of image analysis strategies that mainly differ in their level of accuracy and throughput (for an overview of tools, see Parekh et al. ${ }^{39}$; Fig. 2). Tracing methods tend to delineate individual neuronal extensions, with high accuracy, but typically demand well-contrasted individual neurons. Thus, either isolated neurons or sparsely labelled neuronal networks are warranted. The latter is typically achieved by means of stochastic labelling methods (e.g. Golgi staining or DiI) or transgene mouse models (e.g. Thyl-YFP ${ }^{40}$ or Brainbow mice ${ }^{41}$ ). Tracing is done either manually or semi-automatically, assisted by global image processing operations and/or 
local path finding algorithms. An alternative group of methods to define neuronal morphology rely on global, intensity-based thresholding. The advantage of such methods is that they can be applied easily to sparsely labelled networks, but also to completely stained, dense networks (using pan-neuronal markers, such as $\beta$-III-tubulin or MAP2). Once the neuron is segmented, different metrics can be derived depending on the density of labelled cells. For sparse labelling methods, a fairly simple technique to gauge the complexity of individual neuronal morphology is based on Sholl analysis. In addition, more detailed metrics of single neurons can be obtained such as neurite length and dendritic branching. For pan-labelled neuronal networks, an estimate of these neuron-specific parameters can be given, provided a neuron-specific nuclear counterstaining is available/included. 

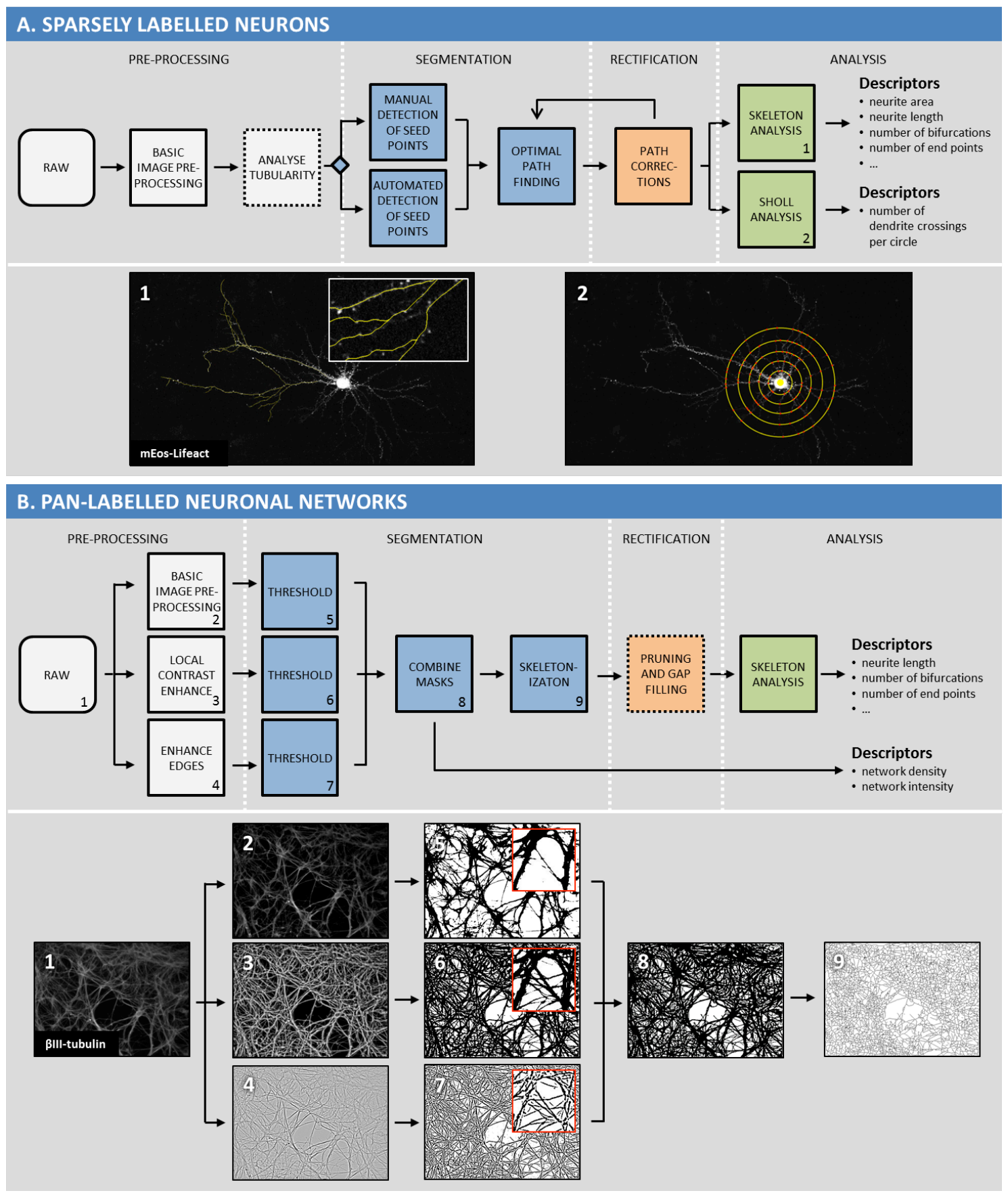

Figure 2 - Morphological analysis of sparsely labelled and pan-labelled neuronal networks

A. To acquire a detailed view of individual neurite length, sparsely labelled neurons can be traced using semiautomated and automated algorithms. The traced neuron can then be subjected to skeleton analysis to derive detailed information about the neuron's morphology, or to Sholl analysis. The latter method describes the complexity of the neuronal morphology by the number of intersections of the neurites with a group of concentric circles drawn around the cell soma. B. This panel shows a multi-tier global segmentation method for analysing pan-labelled neuronal cultures, as implemented in MorphoNeuroNet ${ }^{42}$. A combination of intensity-based $(2,3)$ and edge-based (4) pre-processing algorithms enables the detection of neurites with variable thickness and fluorescence intensity. 


\section{Sparsely labelled neurons.}

Starting from the camera lucida, an optical superposition system that was used to draw the outline of nerve cells by hand, several efforts have been made to generate digital reconstructions of neuronal morphology. The first tools that became available (e.g. Neuron_Morpho ${ }^{43}$ and Neurolucida ${ }^{44}$ ) enabled the manual delineation of neurites in a single plane. Although more recent methods allow the segmentation of neuronal processes in $3 \mathrm{D}$ by delineating $2 \mathrm{D}$ projected images ${ }^{45}$, manual annotation is slow and labour-intensive, and therefore not easily amenable to upscaling.

Although the nomenclature and classification of automated neuron tracing algorithms is not consistent in literature, from an image informatics perspective, we discern global image processing methods, local tracing methods, and algorithms that use a combination of both.

Early attempts to automate the neurite reconstruction process are based on a global intensity threshold, followed by voxel thinning or a medial axis transform to obtain the neurite skele$\operatorname{ton}^{46,47}$. As a result of the global threshold, these methods experience difficulties in the presence of signal inhomogeneities, and the iterative nature of the voxel thinning process is computationally intensive. More recent methods are based on a semi-automatic modus operandi, which relies on local computer-aided identification of putative neurites, in tandem with manual interaction and/or correction. These local exploratory algorithms, also referred to as neuron tracing, better accommodate for gradual changes in neuron morphology and image quality. Various methods have been developed for the local detection of neurite structures. Amongst these, ridge detectors, such as a Hessian filter, which compute a square matrix of second order partial derivatives for every pixel of the image, are used to measure the local tubularity. The directionality of the neurite is obtained by calculating the eigenvectors from the obtained Hessian matrix. The eigenvector with the smallest absolute eigenvalue points in the direction of the vessel (i.e. the direction with the smallest intensity variations). Neuron $\mathrm{J}^{48}$ relies on this algorithm to determine the optimal path (that with the lowest cost) between manually defined start- and endpoints (seeds). This approach is also known as live-wire segmentation. Although NeuronJ was conceived for 2D images, the cost function can readily be extended to 3D by using voxel cubes instead of 2D kernels for the Hessian (as implemented in NeuroMantic ${ }^{49}$ and AutoNeuron for Neurolucida ${ }^{44}$ ). Other implementations to locally reconstruct neuronal morphology rely on the modelling of deformable templates and the iterative addition of structural components (e.g. cylinders) ${ }^{50-52}$. Since these local tracing methods produce one branch at the time, a separate branch point detection method is required to complete the reconstruction ${ }^{53}$. Alternatively, model-free local tracing strategies, such as Rayburst Sampling ${ }^{54}$ and Voxel Scooping ${ }^{55}$, are able to trace multiple branches from a single seed (typically the cell soma). Although these methods enable fully automated segmentation of homogeneously stained neurons, spurious gaps or branches can still occur when the implemented pre-processing steps fail to accurately separate foreground and background. To address this issue, algorithms have been developed to retrospectively attach disconnected branches based on parameters, such as orientation, distance, curvature and intensity ${ }^{56}$. An alternative approach is to directly combine local tracing algorithms with global processing methods to find multiple seed points at critical points (such as terminations, bifurcations and inflections) and to guide the finer-scale tracing process ${ }^{57,58}$. While automation of the neurite tracing process continues to improve, human intervention is often still required to steer the tracing process.

Once obtained, morphological information can be extracted from the segmented neuron. An old, but still widely used method to study segmented neurons is Sholl analysis ${ }^{59}$. This method 
counts how many times the neurites intersect a series of concentric shells that are drawn around the cell soma. Consequently, highly bifurcated neurite networks will return high Sholl values. This tool, while still widely used, has been criticized for its limited sensitivity and inability to correct for branches that cross the same circle multiple times, and those who extend tangentially and do not cross a circle at all. This is why current methods tend to focus more on extracting metrics that can be derived from the backbone, such as neurite length and bifurcation points.

\section{Pan-labelled neuronal networks}

Because neurite tracing relies on the precise delineation of individual neurons, the throughput of this analysis method is generally low. Detailed neuronal models of neurons, however, are very useful to investigate shape/function relations, or in theoretical neurobiology, in which neuronal morphology is used to describe its electrotonic compartmentalization ${ }^{60}$. When a higher throughput is required, global methods can be used to segment multiple neurons in the field of view. Although these methods might lack the precision of neuron tracing in case of signal inhomogeneities in the branches, they are well able to detect general changes in neuronal morphology (e.g. neurite length) in response to compounds that affect neurite outgrowth $^{61}$.

All global segmentation methods rely on binarization (i.e. thresholding) and skeletonization of a pre-processed image (Figure $2 \mathrm{~B})^{62}$. The complexity of the pre-processing steps (apart from those mentioned in 2.1) is what truly discriminates different methods, and this is usually based on the image quality and density of the cell culture. Especially in dense networks, the key is to detect both low and high intensity structures of different sizes. To this end, multiscale or multi-tier object enhancement approaches have been implemented. MorphoNeuroNet $^{42}$ for example, uses a combination of local contrast enhancement and edge detection algorithms (unsharp masking, Laplace filtering) to highlight less intense parts of the neuronal network. A combination of these images after thresholding generates a more complete mask of the neuronal network than any individual image would. Although this binary mask offers a basic measure of the network density, it is often skeletonized to retrieve more detailed parameters, including neurite length and diameter, the number of bifurcations and endpoints. As the resulting skeleton often contains errors (such as spurious gaps or branches), filling and pruning strategies are often used to rectify these retrospectively ${ }^{63}$.

In many neuronal network analyses, a measure of cellular density is calculated as well. Cell or soma segmentation is facilitated in the presence of a nuclear counterstain. Indeed, nuclei are preferred as seeds, because of their well-separated distribution and relatively regular shape (this regularity has recently been challenged; cf. Box 1). Starting from the nuclear boundaries, regions of interest (ROIs) are then grown to detect the soma. 


\section{Box 1 - Nuclear morphology as a novel correlate of neuronal connectivity}

Neuronal nuclei have been shown to be extremely mouldable. They can adopt shapes that range from near spherical to complex and highly folded, and this is correlated with neuronal activity ${ }^{64}$. Nuclear folding has been suggested to be necessary for relaying calcium signals to the nucleus, which is fundamental for proper gene expression ${ }^{1}$. The activitydriven morphological changes of the nucleus are referred to as morphology modulation. Quantification of the internal structure or folding of the nucleus may thus serve as a readout for neuronal connectivity.

Nucleus segmentation is often included in neuronal image analysis pipelines as a starting point for segmenting cell bodies and/or neurites ${ }^{35}$. From segmented nuclei in 2D images, nuclear shape descriptors, such as surface and circularity, can easily be derived using general object enhancement and thresholding procedures. As far as the internal nuclear structure is concerned, phenomena, such as folding, have been addressed far less. Nuclear folds are generally visualized using stains for the nuclear lamina and analysed using procedures that often include manual assessment ${ }^{64,65}$. To describe the internal structure of nuclei in more objective terms, an automatic image analysis procedure has been developed $^{66}$ that quantifies the 3D internal structure of nuclei on the basis of a nuclear lamina stain using three descriptors: mean intensity, skewness and mean curvature. To track nuclear morphological changes over time, Gerlich et al. ${ }^{67}$ developed a technique for fully automated quantification and visualization of surfaces from dynamic 3D fluorescent structures in live cells. 3D surface models were constructed for the nuclear membrane and interpolated over time using a process called morphing. These 4D reconstructions, which allow the quantification of volume changes in the nucleus of live cells, could also serve as an indirect measure of nuclear folding. However, both methods require a complex 4D analysis to achieve a level of accuracy that is not necessary for measuring nuclear folding. To make quantification of nuclear folding amenable to upscaling (highthroughput), we implemented a 2D analysis. In our workflow (Fig. 3), 3D widefield image stacks of lamin-stained neuronal nuclei are Z-projected and nuclei are detected by means of image thresholding followed by a watershed to dissociate neighbouring nuclei. Second, cross-referencing the nuclei with a marker dedicated to neuronal nuclei (e.g. NeuN) allows the selection of neuronal nuclei only, a process that is necessary in cell cultures, which typically consist of neuronal as well as non-neuronal nuclei such as those of astrocytes. Third, the lamin staining is used for segmentation of nuclear folds. A Laplace filter specifically enhances the edges of nuclear folds as well as the edge of the nucleus. To exclude the latter, the ROIs from the initial nuclear segmentation are eroded and only particles lying within the eroded ROIs are identified as folds. For each segmented ROI, the degree of folding is calculated. 


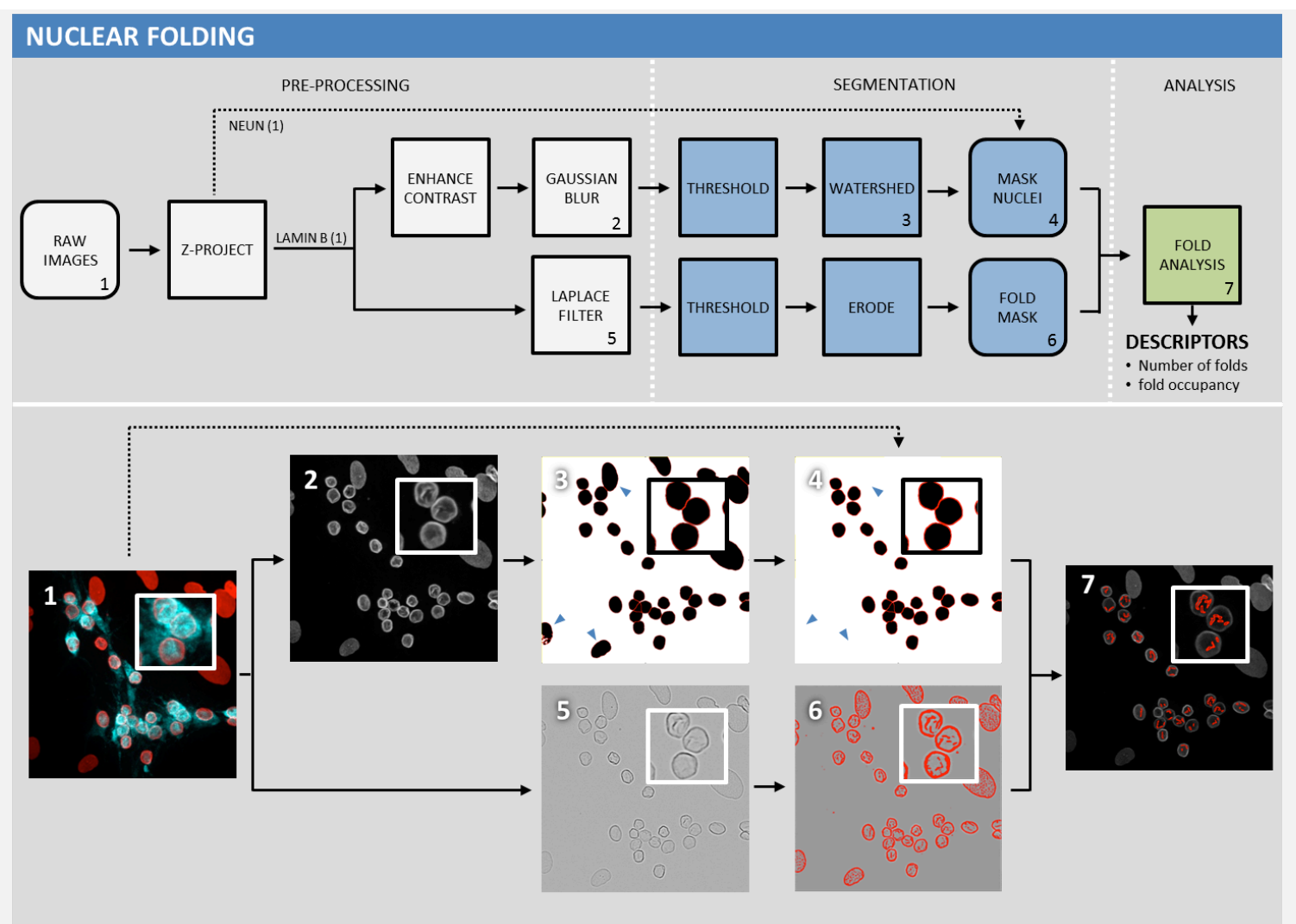

Figure 3 - Quantification of nuclear folding

The percentage of nuclear folding can be determined from images of lamin-stained $($ color $=\ldots$. neuronal nuclei. First, neuronal nuclei are extracted based on a neuronal marker $($ color $=\ldots)(3,4)$. In parallel, a Laplace filter enhances (5) the detection (6) of nuclear folds and edges of the nuclei on lamin-stained images. To identify only the ROIs that represent nuclear folds, the nuclear masks (4) are eroded and only the ROIs that are confined within these regions are detected.

\subsection{Sampling synapses}

Synapses are small structures that are close to or below the diffraction limit $\left(<0.1 \mu \mathrm{m}^{2}\right)$, which is why their detection is often limited to the quantification of diffraction-limited spots or puncta (synapse density). Pan-synaptic labelling is typically achieved by targeting hallmark proteins of the pre- or postsynaptic compartments (e.g. synaptophsyin-I, synapsin and PSD95), although synapses that process specific neurotransmitters can be discerned as well using vesicle- or receptor-specific antibodies (e.g. VAChT, VGAT and GluR). Dendritic spines are more pronounced neuronal substructures that only harbour excitatory synapses ${ }^{68}$, but exhibit different shapes that can be quantified and have been suggested to relate to synaptic health. To visualize spines, the same pan-cellular labelling methods are used as those discussed for analysing the neuronal morphology of sparsely labelled neurons. 


\section{A. SYNAPSES}

PRE-PROCESSING

SEGMENTATION

RECTIFICATION

ANALYSIS
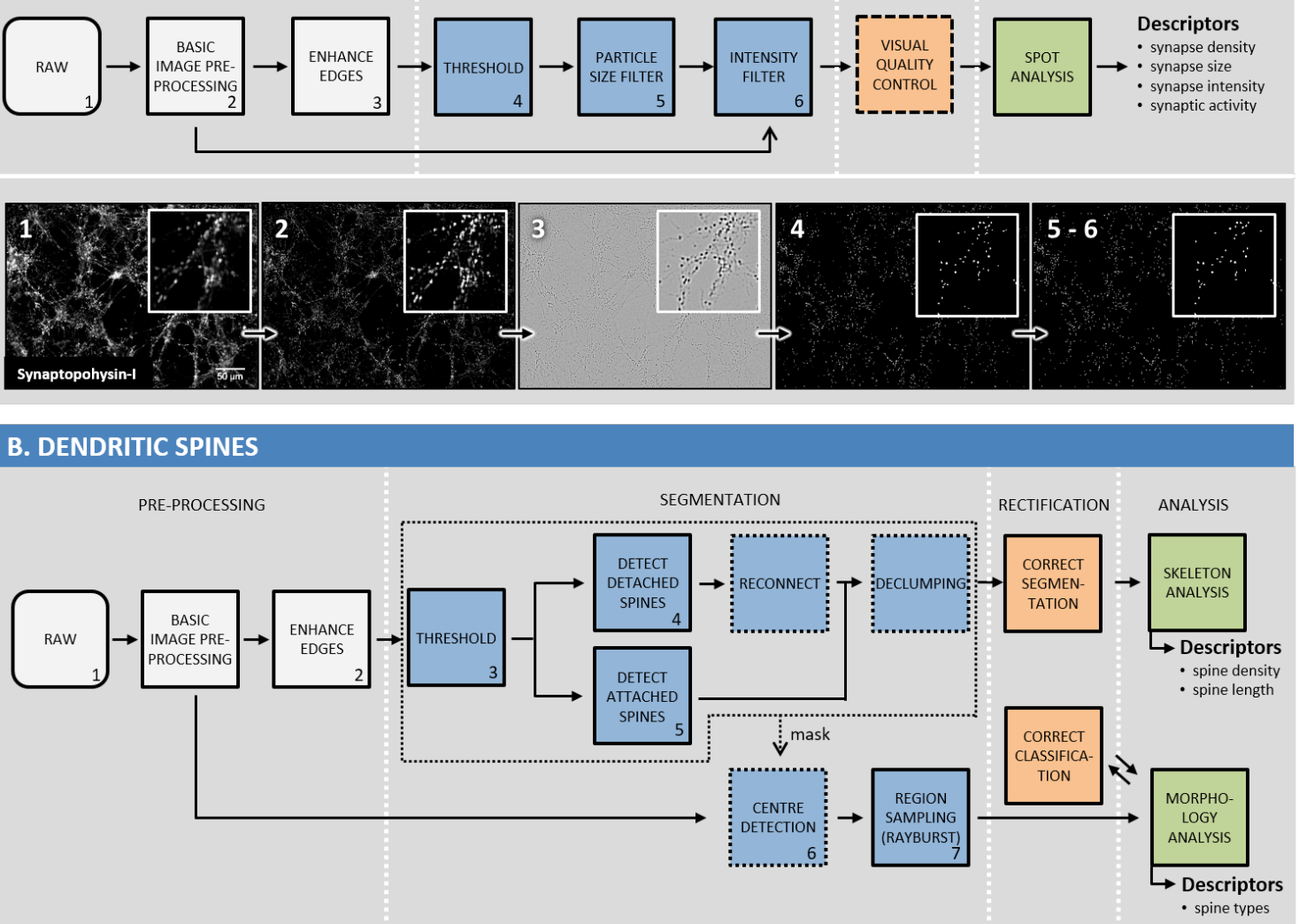

DENDRITIC SPINE SEGMENTATION AND SHAPE ANALYSIS
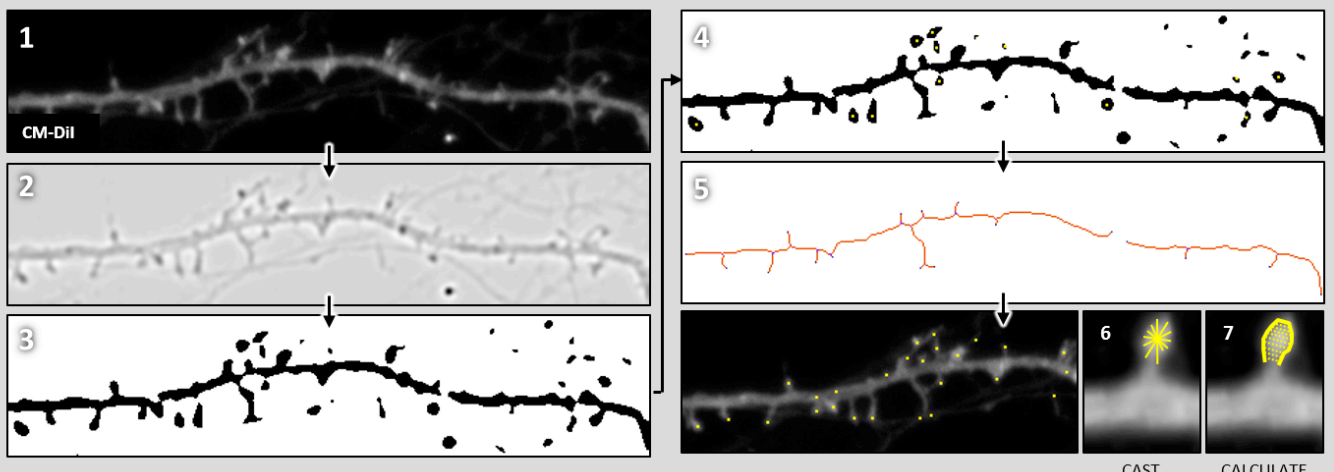

CAST CALCULATE

DENDRITIC SPINE CLASSIFICATION
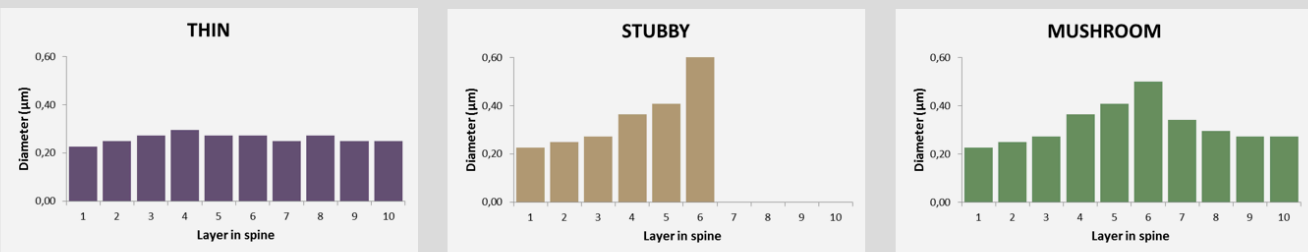

Figure 4 - Image analysis of synapses and dendritic spines 
A. Synapse puncta are extracted by means of spot segmentation (Laplace filter). In a next step, false positives can be eliminated from the resulting image, using intensity and size-based filters. B. In the upper panel, workflows for extracting dendritic spine density and morphology are shown. In the lower panel, the process is shown of a centreline-based segmentation method, followed by Rayburst sampling to estimate the diameter in different layers of the spine. The ratio between the width of the spine head and neck can then be used to classify the spine type (stubby: no neck defined; thin: low ratio; mushroom: high ratio).

\section{Counting synaptic puncta}

Although numerous spot segmentation approaches have been developed ${ }^{69}$, the small size of synapses makes the segmentation process very sensitive to image noise and local variations in contrast (e.g. synaptic structures that display weak signal intensity or the presence of intense background signals originating from the soma or thick dendritic branches). Therefore, instead of more conventional noise filtering methods (cf. section 2.1), advanced denoising strategies (e.g. the wavelet-based algorithm Multi-Scale Variance Stabilizing Transform (MSVST)) have been proposed to enhance threshold-based segmentation of synaptic structures $^{70}$.

To further accommodate for local variations in contrast, local adaptive threshold algorithms, whether or not preceded by blob detectors, such as a Mexican hat or Laplace filters, can be used. In essence, the latter algorithms rely on the assumption that synaptic puncta can be modelled as 2D Gaussian functions. A potential disadvantage of these operators is that the approximate size of the Gaussian should be specified up front. A solution to this is the use of machine-learning algorithms that estimate the size of the kernel ${ }^{34,71}$. As implemented in $\mathrm{SynD}^{34}$, particles with a unique local intensity maximum can be used to generate a datadriven single synapse kernel. Alternative solutions are multiscale spot segmentation approaches ${ }^{72,73}$ or granulometric analysis to "sieve" image objects with structure elements based on their geometry and size ${ }^{74}$.

In a final step, several criteria can be implemented for filtering false positive results. Particle size filtering and intensity cut-offs can be used to separate true synaptic puncta from noise. Other methods also implement distance criteria to exclude particles that are not connected to the neuronal skeleton ${ }^{34}$.

Although there is a limited availability of tools that implement synapse detection, SynD was successfully used in knockout studies aimed at identifying proteins that are involved in synaptic transmission pathways, such as neurotransmitter vesicle fusion ${ }^{75}$ and neurotransmitter receptor trafficking ${ }^{76}$. This tool was later used to evaluate the efficacy of synapto-protective drugs in a micro-fluidics screening platform ${ }^{77}$.

\section{Detection of dendritic spines}

Since dendritic spines are membranous protrusions that form an integral part of the neurite network, their segmentation is usually part of neuronal network segmentation approaches. Therefore, most tools that have been developed for the detection of dendritic spines rely on or have built-in neurite tracing tools (e.g. Neuronstudio ${ }^{54}$, AutoSpine ${ }^{78}$ ).

As for segmentation of the previously discussed morphological parameters, a simple global intensity threshold is inadequate to segment spines, since this approach fails to accurately detect faint or thin spines without distorting the shape of more intense spines. To address this issue, edge enhancers (e.g. Laplace filtering or unsharp masking ${ }^{79}$ ) and local adaptive threshold algorithms ${ }^{80,81}$ are used. In contrast to threshold-based methods, another category of spine segmentation algorithms uses a curvilinear structure detector ${ }^{82}$. This filter, used in many med- 
ical image-processing algorithms (e.g. for detecting blood vessels, airways or bones), delineates the dendritic backbones directly on the original image by treating them as 2D line objects. A similar method is then used to detect the centrelines of dendritic spines. After segmentation and skeletonization, most dendritic spines are usually identified as protrusions (Figure 4A $)^{46,79,80}$. Some spines, however, become detached in the segmentation process and should be reassigned, e.g. based on the distance from the backbone and on size criteria ${ }^{79}$. More advanced methods rely on a classifier, built from a library of isolated spines ${ }^{82}$.

Although centreline extraction-based approaches offer a reasonable quantification of lateral spines, the limited axial resolution of microscopes makes them unreliable for quantifying spines that are oriented orthogonal to the imaging plane. Therefore, most centreline-based algorithms estimate the spine density from maximum intensity projected images, which leads to a substantial underestimation of spine densities ${ }^{79,80,82}$. While variations in the skeletonization algorithm have led to increased accuracy of spine detection in $3 \mathrm{D}^{46,83}$, these algorithms are computationally expensive. Model-based algorithms, such as voxel clustering ${ }^{81}$ and the marching cubes algorithm ${ }^{84}$, are faster alternatives that identify spines based on a trained classifier. In addition, 3D Gabor wavelets have recently been proposed as a fast method for detecting dendritic spines by clustering candidate voxels according to the response to the wavelet transform ${ }^{85}$.

None of the existing algorithms are error-free. One common problem is that neighbouring spines are merged on the segmented images as a result of low image resolution or incorrect thresholding. To solve this, one can rely on the fact that voxel intensities are naturally brighter at the centre of spines and dimmer at the edges. Clumped spines can then be delimited based on their 3D intensity vector gradients ${ }^{81}$. Other methods rely on 3D shape analysis to automatically categorize spines into single spines or touching spines ${ }^{86}$.

\section{Determining spine morphology}

In centreline extraction-based methods, morphology determination is mainly limited to quantifying the length of the segmented dendritic spines. Since small structures, such as dendritic spines, comprise only a few voxels at maximal imaging resolution, quantization errors due to the finite voxel representation in digital images can be significant. Rayburst sampling was introduced to allow more reliable morphometric studies of dendritic spines. This is done by casting a multidirectional core of rays from an interior point (i.e. the centre of mass of the spine) to the spine surface, allowing precise sampling of its anisotropic and irregularly shaped structure. As the ray pattern is casted with sub-voxel accuracy using interpolated pixel intensity values, quantization errors are minimized. Once the contours of the spine are sampled, the spine diameter is calculated for different layers between the spine head and spine neck (Figure 4B). The aspect ratio and the width of the head are then used to resolve the final spine types. Rayburst sampling has been successfully used to detect a decrease in spine volume and dendrite diameter in mouse models for Huntington's disease $\left(\mathrm{R} 6 / 2^{36}\right)$ and Alzheimer's disease $\left(\mathrm{TG} 2576^{87}\right.$ ). In addition to its original implementation in NeuronStudio ${ }^{54}$, the algorithm was also adopted by AutoSpine (part of Neurolucida $360^{44}$ ) and FilamentTracer ${ }^{88}$. 


\section{SIZING THE WAVES OF ACTIVITY: QUANTIFYING CALCIUM FLUXES}

\subsection{Visualizing electrical activity}

Electrical activity exhibited by neurons can be visualized under the microscope using membrane voltage sensors. Classical voltage sensors such as potential sensitive aminonaphthylethenylpyridinium (ANEP) dyes display a spectral shift upon a change in voltage across the membrane ${ }^{31}$; more recently developed genetically encoded sensors, such as $\mathrm{FlaSh}^{89}$, Electric $\mathrm{Pk}^{90}$ or ArcLight ${ }^{32,91}$, change intensity with voltage. Despite rapid developments in the field ${ }^{92}$, voltage sensors still do not cover a very high dynamic range and typically have to be measured very fast (up to $60 \mathrm{kHz}$ ). This is why electrical activity is still most often measured indirectly, by gauging calcium fluctuations ${ }^{93,94}$. The high dynamic range of most calcium sensors allows visualizing electrical activity on a conventional fluorescence microscope at the single-neuron scale, albeit at lower temporal resolution (typically 2-4 Hz) than voltage imaging. Non-ratiometric calcium probes, such as Fluo4-AM, display an increase in fluorescence intensity upon calcium binding, while ratiometric probes like Fura-2 exhibit a shift in excitation or emission spectra, allowing precise measurements of intracellular calcium concentration, not biased by uneven dye loading. In addition to synthetic calcium probes, genetically encoded sensors like chameleons or GCaMPs have emerged over the last years ${ }^{30}$. These sensors allow long-term follow-up of neuronal activity and their expression can be limited to neurons, e.g. when driven by a synapsin promoter. Also, their spatial localization can be confined to e.g. synaptic compartments, when fused to synaptic proteins.

\subsection{Measuring calcium fluxes}

Reliable quantification of dynamic calcium recordings requires integrated image and signal analysis. The workflow of such an analysis is depicted in figure 5 (upper panel), together with the output from a Fluo-4AM recording of spontaneous activity in a primary hippocampal culture of 7 days in vitro (DIV, lower panel).

To allow proper assessment of intercellular synchronicity of calcium oscillations, it is essential that individual neurons be properly segmented. This issue is resolved by including a nuclear label since the somas are the most abundant calcium domains. If neuron-specific nuclear tags are available (e.g. nuclear-localized fluorescent proteins expressed under a synapsin promoter), the analysis can immediately proceed to the signal analysis stage. However, synthetic nuclear indicators load all cells and require discrimination between the segmented neurons and astrocytes in the field of view. This can be achieved by exposing the cultures to a high concentration of glutamate, since neurons are known to respond with a very fast and prolonged increase in intracellular calcium, while astrocytes exhibit a delayed and transient calcium wave ${ }^{95}$. The first step following the extraction of calcium traces from the segmented cells is to define the glutamate addition point (typically the maximum signal). Then, two measures can be used to classify the cellular responses. First, the rise time can be used to detect delayed and slow responses of non-neuronal cells. Second, non-neuronal cells can be discarded based on their relative faster loss in mean fluorescence intensity after glutamate addition. 


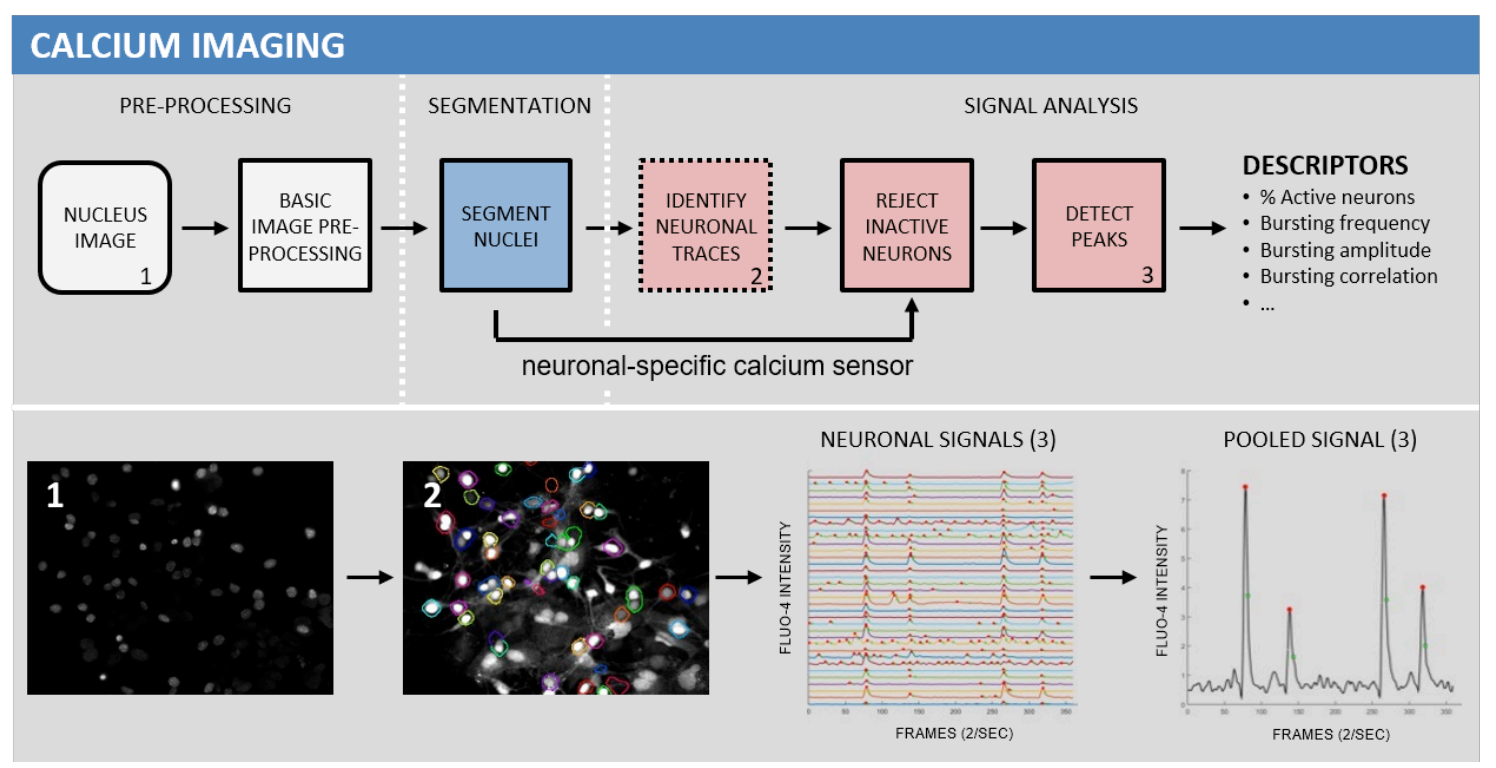

Figure 5 - Workflow for analysing calcium recordings from neuronal cultures. The upper panel shows image and signal analysis steps to extract numerical data from calcium recordings, while the lower panel contains output from a primary hippocampal culture showing both synchronized (corresponding to peaks in the pooled signal) and asynchronous calcium bursts.

Similar pre-processing operations to those explained for 2D images (e.g. background subtraction and smoothing) are then performed on the $1 \mathrm{D}$ neuronal signals. Inactive neurons are identified based on a signal cut-off and rejected from the downstream analysis. Noise-tolerant peak detection on active neurons returns the location (burst frequency) and amplitude of each peak, as well as the average $50 \%$ decay time. Peaks displaying a decay time above a usersupplied maximum are discarded from the analysis and are reported as the number of long decays. Readouts originate from the rejection of inactive neurons ( $\%$ active neurons) or from peak detection on individual (frequency, amplitude, decay time) or pooled (frequency of synchronized bursts) signals. However, one of the most sensitive readouts for quantifying the correlation of calcium oscillations across individual neurons is the burst correlation or synchronicity score ${ }^{19}$.

The proposed image and signal analysis pipeline allows quantifying the effects of chronic pharmacological or genetic treatments on neuronal connectivity with great sensitivity ${ }^{18}$. For instance, it was shown that deprivation of nerve growth factor (NGF) impaired the synchronization of neuronal activity while increased trophic support by a feeder layer of astrocytes enhanced network formation. Additionally, division of a recording into 2 or 3 stretches allows the evaluation of the acute responses to pharmacological treatments. In this context, it was shown that synchronized network activity is mediated by the NMDA receptor, as NMDA receptor antagonists decreased the synchronicity score. Calcium imaging of in vitro network activity has also been used to study epilepsy by application of the convulsive drug 4aminopyridine and low magnesium ${ }^{96}$. Using an experimental in vitro model of traumatic brain injury, the neuronal response to subsequent glutamate stimulation has also been studied with calcium imaging ${ }^{97}$. 


\section{CONCLUSION AND PERSPECTIVES}

In this work we have given an overview of the image analysis algorithms that are used to investigate neuronal connectivity in cell cultures. We discussed the extraction of morphological features, such as the analysis of neuronal morphology and synapses, as well as the measurement of functional parameters used in calcium activity-related imaging studies.

When addressing neuronal morphology, a clear trade-off should be made between accuracy and throughput, and this has to be aligned with the labelling procedure. Whereas neuronal tracing provides an accurate representation of sparsely labelled neurons, it currently still demands manual intervention to rectify segmentation errors. A machine-learning approach that is trained using a manually delineated dataset, has recently been proposed to reduce the proofreading time by only highlighting the reconstructions with lowest confidence ${ }^{98}$. Further elaboration on this approach may lead to a user-independent self-learning algorithm, such as SmartTracing $^{99}$, in which there is no need for a sample dataset. On the other hand global segmentation algorithms can be used to delineate neurons and pan-labelled, dense networks in a fully automated mode, albeit with lower accuracy. Recent developments are aimed at combining both global and local segmentation methods to develop fully automated tracing methods that are robust to staining imperfections and noise ${ }^{57}$. Although early neuronal tracing algorithms were limited to 2D, 3D tracing algorithms are currently fine-tuned in such a way that they can be used to analyse stained neurons in neuronal slices, or even in the intact cleared brain ${ }^{100}$. To this end, similar stochastic labelling procedures can be used for the sparse labelling of single neurons. Alternatively, more refined labelling strategies (e.g. based on GFP-expressing neurotropic viruses ${ }^{101}$ ) that allow trans-synaptic tracing of neurons open doors for more detailed connectome studies. This work further shows that numerous, sometimes redundant, approaches (described in literature) are currently employed to analyse neuronal morphology, making it difficult to select the best method for a given dataset ${ }^{102}$. In order to compare the accuracy and the computational efficiency of these different methods, the BigNeuron project was launched in March $2015^{12}$. The major goal of this project is to enhance neuron reconstruction by bench-testing multiple algorithms against a large neuron dataset based on the experience of different research groups around the world.

Synapses are analysed by direct labelling of proteins involved in synaptic processing, or by assessing the density and morphology of dendritic spines. Although synaptic puncta are easily extracted using blob detectors, pre- and post-processing is often necessary to discriminate the true synaptic puncta from noise. Whereas a count of synapses offers an estimate of the number of synaptic proteins, a colocalisation analysis of pre- and postsynaptic labels (e.g. VGluT and PSD95) can be performed to define synaptic partners ${ }^{103,104}$. In addition, FM dyes can be used to selectively stain the presynaptic membrane of living cells to monitor neurotransmitter release and reuptake over time ${ }^{70}$. The extension of synapse segmentation to $3 \mathrm{D}$ is limited by the spatial resolution of confocal microscopes in the axial direction. A solution to this issue is to computationally reconstruct serial ultrathin sections, known as array tomography ${ }^{105}$. Alternatively, 3D superresolution imaging (e.g. 3D STORM ${ }^{106}$ ) can be used for volumetric imaging of synapses without the requirement of sectioning.

From an image informatics perspective, dendritic spines are more difficult to detect compared to synapses. This is because the segmentation process has to accommodate for the irregular and variable shape of spines, compared to the more consistent spot pattern that is found for synapse markers. Despite the development of numerous workflows that incorporate parallel 
analysis lines to increase the detection accuracy of spines, fully automated detection of spines is still a challenge. Similarly, classifying spine morphology requires the input of a human operator for reasons of quality control. Although most image processing algorithms are used to analyse small stacks of in vitro recordings, 3D dendritic spine analysis has also been carried out in tissue slice cultures ${ }^{87}$ and in vivo recordings ${ }^{107}$. Tracking the changes in dendritic spine density and morphology in living animals would not only allow real-time monitoring of the acute effects of drug treatments, but also enable direct correlation of neuronal connectivity parameters with cognitive and behavioural characteristics.Calcium imaging is a valuable tool in the emerging field of iPSC technology to characterize iPSC-derived neurons and to detect phenotypes in patient-derived cultures ${ }^{108-111}$. Although calcium imaging studies are mostly performed on monocultures, a direct extension of such experiments would be to shift to the co-cultivation of differentially labelled neuronal cultures. This enables the study of cell-cell interactions on calcium bursting behaviour, which might be of interest to investigate the effect of trans-synaptically transmitted toxic proteins ${ }^{112}$. In addition, calcium imaging can be combined with optogenetics ${ }^{113}$ or photostimulation ${ }^{114}$, so as to perturb specific cells (or even subcellular compartments) and monitor response within a multicellular context. Closing the loop between optical readouts and the generation of these stimuli (i.e. by real-time generation of stimuli based on live image analysis) will provide a powerful strategy to study cause-andeffect relationships in neural circuitry ${ }^{115}$. Although this discussion was limited to calcium imaging of in vitro neuronal networks, obviously such measurements can be expanded to live animals. However, this brings about an additional layer of complexity and imposes challenges, such as correction for motion artefacts and discrimination of calcium signals that originate from different layers in the tissue ${ }^{116}$. Tackling these issues, however, will lead to the emergence of further advanced experimental setups, such as those in which mice are subjected to virtual reality systems to study their spatial navigation ${ }^{117}$.

In conclusion, a lot of work has been done to automate the quantification of morphological and functional features of neuronal networks. The ultimate goal of these image analysis algorithms is to provide an accurate, fully automated assessment of neuronal network status. Although there are still challenges to be met in this respect, image informatics is an essential component of image-based screening assays. Considering (?) new methods for tissue preparation and labelling, continuing advances in microscopic imaging systems and further development of image analysis tools are/will be essential to extract meaningful data from microscopic images. 


\section{ACKNOWLEDGEMENTS}

This work was supported by the Agency for Innovation by Science and Technology in Flanders (IWT Baekeland fellowship IWT_140775, O\&O IWT_150003), the Flemish Institute for Scientific Research (FWO PhD Fellowship 11ZF116N) and the University of Antwerp (UA_29267,UA_29256). We further would like to acknowledge Dr. Steffen Jaensch and Dr. Andreas Ebneth for their valuable comments and discussions.

\section{LITERATURE}

1. Bading, H. Nuclear calcium signalling in the regulation of brain function. Nat Rev Neurosci 14, 593-608 (2013).

2. Alvarez, V. a \& Sabatini, B. L. Anatomical and physiological plasticity of dendritic spines. Annu. Rev. Neurosci. 30, 79-97 (2007).

3. Lin, Y.-C. \& Koleske, A. J. Mechanisms of synapse and dendrite maintenance and their disruption in psychiatric and neurodegenerative disorders. Annu. Rev. Neurosci. 33, 349-378 (2010).

4. Penzes, P., Cahill, M. E., Jones, K. A., VanLeeuwen, J. E. \& Woolfrey, K. M. Dendritic spine pathology in neuropsychiatric disorders. Nat Neurosci 14, 285-293 (2011).

5. Cai, D., Cohen, K. B., Luo, T., Lichtman, J. W. \& Sanes, J. R. Improved tools for the Brainbow toolbox. Nat Methods 10, 540-547 (2013).

6. Chen, J. L., Andermann, M. L., Keck, T., Xu, N.-L. \& Ziv, Y. Imaging neuronal populations in behaving rodents: paradigms for studying neural circuits underlying behavior in the mammalian cortex. J. Neurosci. 33, 17631-40 (2013).

7. Dombeck, D. A., Khabbaz, A. N., Collman, F., Adelman, T. L. \& Tank, D. W. Imaging large-scale neural activity with cellular resolution in awake, mobile mice. Neuron 56, 43-57 (2007).

8. Nemoto, T. Development of novel two-photon microscopy for living brain and neuron. Microscopy 63, (2014).

9. Kim, S.-Y., Chung, K. \& Deisseroth, K. Light microscopy mapping of connections in the intact brain. Trends Cogn. Sci. 17, 596-9 (2013).

10. The Allen Institute. at $<$ http://alleninstitute.org/>

11. The Connectome Project. at $<$ http://cbs.fas.harvard.edu/science/connectome-project $>$

12. Peng, H., Roysam, B. \& Ascoli, G. A. Automated image computing reshapes computational neuroscience. BMC Bioinformatics 14, 293 (2013). 
13. Cho, S., Wood, A. \& Bowlby, M. R. Brain slices as models for neurodegenerative disease and screening platforms to identify novel therapeutics. Curr. Neuropharmacol. 5, 19-33 (2007).

14. Cohen, E., Ivenshitz, M., Amor-Baroukh, V., Greenberger, V. \& Segal, M. Determinants of spontaneous activity in networks of cultured hippocampus. Brain Res 1235, 21-30 (2008).

15. Dotti, C. G., Sullivan, C. A. \& Banker, G. A. The establishment of polarity by hippocampal neurons in culture. J Neurosci 8, 1454-1468 (1988).

16. Fletcher, T. L., De Camilli, P. \& Banker, G. Synaptogenesis in hippocampal cultures: evidence indicating that axons and dendrites become competent to form synapses at different stages of neuronal development. J Neurosci 14, 6695-6706 (1994).

17. Papa, M., Bundman, M. C., Greenberger, V. \& Segal, M. Morphological analysis of dendritic spine development in primary cultures of hippocampal neurons. $J$ Neurosci 15, 1-11 (1995).

18. Verstraelen, P. et al. Pharmacological characterization of cultivated neuronal networks: relevance to synaptogenesis and synaptic connectivity. Cell. Mol. Neurobiol. 34, 757-76 (2014).

19. Cornelissen, F. et al. Quantitation of chronic and acute treatment effects on neuronal network activity using image and signal analysis: toward a high-content assay. $J$. Biomol. Screen. 18, 807-19 (2013).

20. Takahashi, K. \& Yamanaka, S. Induction of pluripotent stem cells from mouse embryonic and adult fibroblast cultures by defined factors. Cell 126, 663-676 (2006).

21. Imamura, K. \& Inoue, H. Research on neurodegenerative diseases using induced pluripotent stem cells. Psychogeriatrics 12, 115-119 (2012).

22. Harrill, J. A., Robinette, B. L., Freudenrich, T. \& Mundy, W. R. Use of high content image analyses to detect chemical-mediated effects on neurite sub-populations in primary rat cortical neurons. Neurotoxicology 34, 61-73 (2013).

23. Popova, D. \& Jacobsson, S. O. A fluorescence microplate screen assay for the detection of neurite outgrowth and neurotoxicity using an antibody against betaIIItubulin. Toxicol Vitr. 28, 411-418 (2014).

24. Sirenko, O., Hesley, J., Rusyn, I. \& Cromwell, E. F. High-content high-throughput assays for characterizing the viability and morphology of human iPSC-derived neuronal cultures. Assay Drug Dev Technol 12, 536-547 (2014). 
25. y Cajal, S. R. Sur la structure de l'écorce cérébrale de quelques mammifères. (Typ. de Joseph van In \& Cie.; Aug. Peeters, lib, 1891).

26. Sala, C. \& Segal, M. Dendritic spines: the locus of structural and functional plasticity. Physiol Rev 94, 141-188 (2014).

27. Dent, E. W., Merriam, E. B. \& Hu, X. The dynamic cytoskeleton: backbone of dendritic spine plasticity. Curr Opin Neurobiol 21, 175-181 (2011).

28. Lai, K. O. \& Ip, N. Y. Structural plasticity of dendritic spines: the underlying mechanisms and its dysregulation in brain disorders. Biochim Biophys Acta 1832, 2257-2263 (2013).

29. Maiti, P., Manna, J. \& McDonald, M. P. Merging advanced technologies with classical methods to uncover dendritic spine dynamics: A hot spot of synaptic plasticity. Neurosci Res 96, 1-13 (2015).

30. Broussard, G. J., Liang, R. \& Tian, L. Monitoring activity in neural circuits with genetically encoded indicators. Front Mol Neurosci 7, 97 (2014).

31. Fluhler, E., Burnham, V. G. \& Loew, L. M. Spectra, membrane binding, and potentiometric responses of new charge shift probes. Biochemistry 24, 5749-5755 (1985).

32. Jin, L. et al. Single action potentials and subthreshold electrical events imaged in neurons with a fluorescent protein voltage probe. Neuron 75, 779-785 (2012).

33. Paredes, R. M., Etzler, J. C., Watts, L. T., Zheng, W. \& Lechleiter, J. D. Chemical calcium indicators. Methods 46, 143-151 (2008).

34. Schmitz, S. K. et al. Automated analysis of neuronal morphology, synapse number and synaptic recruitment. J. Neurosci. Methods 195, 185-193 (2011).

35. Meijering, E. Neuron tracing in perspective. Cytom. Part A 77, 693-704 (2010).

36. Heck, N., Betuing, S., Vanhoutte, P. \& Caboche, J. A deconvolution method to improve automated 3D-analysis of dendritic spines: Application to a mouse model of Huntington's disease. Brain Struct. Funct. 217, 421-434 (2012).

37. Sarder, P. \& Nehorai, a. Deconvolution methods for 3-D fluorescence microscopy images. IEEE Signal Process. Mag. 23, 32-45 (2006).

38. Zhang, B., Fadili, J. M. \& Starck, J. Wavelets, Ridgelets, and Curvelets for Poisson Noise Removal. Image (Rochester, N.Y.) 17, 1093-1108 (2008). 
39. Parekh, R. \& Ascoli, G. a. Neuronal Morphology Goes Digital: A Research Hub for Cellular and System Neuroscience. Neuron 77, 1017-1038 (2013).

40. Feng, G., Mellor, R. \& Bernstein, M. Imaging neuronal subsets in transgenic mice expressing multiple spectral variants of GFP. Neuron 28, 41-51 (2000).

41. Livet, J. et al. Transgenic strategies for combinatorial expression of fluorescent proteins in the nervous system. Nature 450, 56-62 (2007).

42. Pani, G. et al. MorphoNeuroNet: an automated method for dense neurite network analysis. Cytometry. A 85, 188-99 (2014).

43. Brown, K. M., Donohue, D. E., D’Alessandro, G. \& Ascoli, G. A. A cross-platform freeware tool for digital reconstruction of neuronal arborizations from image stacks. Neuroinformatics 3, 343-360 (2005).

44. Neurolucida. at $<$ http://www.mbfbioscience.com/neurolucida $>$

45. Peng, H. et al. Virtual finger boosts three-dimensional imaging and microsurgery as well as terabyte volume image visualization and analysis. Nat. Commun. 5, 4342 (2014).

46. Koh, I. Y. Y., Lindquist, W. B., Zito, K., Nimchinsky, E. A. \& Svoboda, K. An Image Analysis Algorithm for Dendritic Spines. Neural Comput. 14, 1283-1310 (2002).

47. Wearne, S. L. et al. New techniques for imaging, digitization and analysis of threedimensional neural morphology on multiple scales. Neuroscience 136, 661-680 (2005).

48. Meijering, E. et al. Design and validation of a tool for neurite tracing and analysis in fluorescence microscopy images. Cytometry. A 58, 167-176 (2004).

49. Myatt, D. R., Hadlington, T., Ascoli, G. a. \& Nasuto, S. J. Neuromantic - from SemiManual to Semi-Automatic Reconstruction of Neuron Morphology. Front. Neuroinform. 6, 1-14 (2012).

50. Schmitt, S., Evers, J. F., Duch, C., Scholz, M. \& Obermayer, K. New methods for the computer-assisted 3-D reconstruction of neurons from confocal image stacks. Neuroimage 23, 1283-1298 (2004).

51. Zhao, T. et al. Automated reconstruction of neuronal morphology based on local geometrical and global structural models. Neuroinformatics 9, 247-261 (2011).

52. Al-Kofahi, K. a. et al. Rapid automated three-dimensional tracing of neurons from confocal image stacks. IEEE Trans. Inf. Technol. Biomed. 6, 171-187 (2002). 
53. Al-Kofahi, Y. et al. Improved detection of branching points in algorithms for automated neuron tracing from 3D confocal images. Cytom. Part A 73, 36-43 (2008).

54. Rodriguez, A., Ehlenberger, D. B., Hof, P. R. \& Wearne, S. L. Rayburst sampling, an algorithm for automated three-dimensional shape analysis from laser scanning microscopy images. Nat. Protoc. 1, 2152-2161 (2006).

55. Rodriguez, A., Ehlenberger, D. B., Hof, P. R. \& Wearne, S. L. Three-dimensional neuron tracing by voxel scooping. J. Neurosci. Methods 184, 169-175 (2009).

56. Chothani, P., Mehta, V. \& Stepanyants, A. Automated tracing of neurites from light microscopy stacks of images. Neuroinformatics 9, 263-278 (2011).

57. Peng, H., Long, F. \& Myers, G. Automatic 3D neuron tracing using all-path pruning. Bioinformatics 27, 239-247 (2011).

58. Xie, J., Zhao, T., Lee, T., Myers, E. \& Peng, H. Automatic neuron tracing in volumetric microscopy images with anisotropic path searching. Lect. Notes Comput. Sci. (including Subser. Lect. Notes Artif. Intell. Lect. Notes Bioinformatics) 6362 LNCS, 472-479 (2010).

59. Binley, K. E., Ng, W. S., Tribble, J. R., Song, B. \& Morgan, J. E. Sholl analysis: A quantitative comparison of semi-automated methods. J. Neurosci. Methods 225, 65-70 (2014).

60. Costa, L. D. F. et al. A shape analysis framework for neuromorphometry. Network 13, 283-310 (2002).

61. Pool, M., Thiemann, J., Bar-Or, A. \& Fournier, A. E. NeuriteTracer: A novel ImageJ plugin for automated quantification of neurite outgrowth. J. Neurosci. Methods 168, 134-139 (2008).

62. Ho, S.-Y. et al. NeurphologyJ: an automatic neuronal morphology quantification method and its application in pharmacological discovery. BMC Bioinformatics 12, 230 (2011).

63. Narro, M. L. et al. NeuronMetrics: Software for semi-automated processing of cultured neuron images. Brain Res. 1138, 57-75 (2007).

64. Wittmann, M. et al. Synaptic activity induces dramatic changes in the geometry of the cell nucleus: interplay between nuclear structure, histone $\mathrm{H} 3$ phosphorylation, and nuclear calcium signaling. J. Neurosci. 29, 14687-14700 (2009).

65. Lammerding, J. et al. Lamins A and C but not lamin B1 regulate nuclear mechanics. $J$. Biol. Chem. 281, 25768-25780 (2006). 
66. Righolt, C. H., van't Hoff, M. L. R., Vermolen, B. J., Young, I. T. \& Raz, V. Robust nuclear lamina-based cell classification of aging and senescent cells. Aging (Albany NY) 3, 1192 (2011).

67. Gerlich, D., Beaudouin, J., Gebhard, M., Ellenberg, J. \& Eils, R. Four-dimensional imaging and quantitative reconstruction to analyse complex spatiotemporal processes in live cells. Nat. Cell Biol. 3, 852-855 (2001).

68. McKinney, R. A. Excitatory amino acid involvement in dendritic spine formation, maintenance and remodelling. J. Physiol. 588, 107-116 (2010).

69. Meijering, E. Cell Segmentation: 50 Years Down the Road. 29, 140-145 (2012).

70. Fan, J., Xia, X., Li, Y., Dy, J. G. \& Wong, S. T. C. A quantitative analytic pipeline for evaluating neuronal activities by high-throughput synaptic vesicle imaging.

Neuroimage 62, 2040-2054 (2012).

71. Feng, L., Zhao, T. \& Kim, J. Improved synapse detection for mGRASP-assisted brain connectivity mapping. Bioinformatics 28, 25-31 (2012).

72. Bretzner, L. \& Lindeberg, T. Feature tracking with automatic selection of spatial scales. Comput. Vis. Image Underst. 71, 385-392 (1998).

73. De Vos, W. H., Van Neste, L., Dieriks, B., Joss, G. H. \& Van Oostveldt, P. High content image cytometry in the context of subnuclear organization. Cytometry. A 77, 64-75 (2010).

74. Prodanov, D., Heeroma, J. \& Marani, E. Automatic morphometry of synaptic boutons of cultured cells using granulometric analysis of digital images. J. Neurosci. Methods 151, 168-177 (2006).

75. Meijer, M. et al. Munc18-1 mutations that strongly impair SNARE-complex binding support normal synaptic transmission. EMBO J. 31, 2156-2168 (2012).

76. Nair, R. et al. Neurobeachin regulates neurotransmitter receptor trafficking to synapses. J. Cell Biol. 200, 61-80 (2013).

77. Deleglise, B. et al. Synapto-Protective Drugs Evaluation in Reconstructed Neuronal Network. PLoS One 8, 1-9 (2013).

78. AutoSpine. at $<$ http://www.mbfbioscience.com/autospine $>$

79. Bai, W., Zhou, X., Ji, L., Cheng, J. \& Wong, S. T. C. Automatic dendritic spine analysis in two-photon laser scanning microscopy images. Cytom. Part A 71, 818-826 (2007). 
80. Cheng, J. et al. A novel computational approach for automatic dendrite spines detection in two-photon laser scan microscopy. J. Neurosci. Methods 165, 122-134 (2007).

81. Rodriguez, A., Ehlenberger, D. B., Dickstein, D. L., Hof, P. R. \& Wearne, S. L. Automated three-dimensional detection and shape classification of dendritic spines from fluorescence microscopy images. PLoS One 3, (2008).

82. Yong, Z. et al. Automated spine detection using curvilinear structure detector and LDA classifier. in 2007 4th IEEE International Symposium on Biomedical Imaging: From Nano to Macro - Proceedings 36, 528-531 (Elsevier Inc., 2007).

83. Janoos, F. et al. Robust 3D reconstruction and identification of dendritic spines from optical microscopy imaging. Med. Image Anal. 13, 167-179 (2009).

84. Li, Q. et al. A novel surface-based geometric approach for $3 \mathrm{~d}$ dendritic spine detection from multi-photon excitation microscopy images. Proc. - 2009 IEEE Int. Symp.

Biomed. Imaging From Nano to Macro, ISBI 2009 1255-1258 (2009). doi:10.1109/ISBI.2009.5193290

85. Shi, P., Huang, Y. \& Hong, J. Automated three-dimensional reconstruction and morphological analysis of dendritic spines based on semi-supervised learning. Biomed. Opt. Express 5, 1541-53 (2014).

86. Li, Q. \& Deng, Z. A surface-based 3-D dendritic spine detection approach from confocal microscopy images. IEEE Trans. Image Process. 21, 1223-1230 (2012).

87. Luebke, J. I. et al. Dendritic vulnerability in neurodegenerative disease: Insights from analyses of cortical pyramidal neurons in transgenic mouse models. Brain Struct. Funct. 214, 181-199 (2010).

88. FilamentTracer. at $<\mathrm{http}: / / \mathrm{www}$.andor.com/scientific-software/imaris-frombitplane/filamenttracer $>$

89. Siegel, M. S. \& Isacoff, E. Y. A genetically encoded optical probe of membrane voltage. Neuron 19, 735-741 (1997).

90. Barnett, L., Platisa, J., Popovic, M., Pieribone, V. A. \& Hughes, T. A fluorescent, genetically-encoded voltage probe capable of resolving action potentials. PLoS One 7, e43454 (2012).

91. Piao, H. H., Rajakumar, D., Kang, B. E., Kim, E. H. \& Baker, B. J. Combinatorial mutagenesis of the voltage-sensing domain enables the optical resolution of action potentials firing at $60 \mathrm{~Hz}$ by a genetically encoded fluorescent sensor of membrane potential. J Neurosci 35, 372-385 (2015). 
92. Jin, L. et al. in Imaging the Brain with Optical Methods 27-43 (Springer, 2010).

93. Herzog, N., Shein-Idelson, M. \& Hanein, Y. Optical validation of in vitro extracellular neuronal recordings. J Neural Eng 8, 56008 (2011).

94. Smetters, D., Majewska, a \& Yuste, R. Detecting action potentials in neuronal populations with calcium imaging. Methods 18, 215-221 (1999).

95. Pickering, M., Pickering, B. W., Murphy, K. J. \& O’Connor, J. J. Discrimination of cell types in mixed cortical culture using calcium imaging: A comparison to immunocytochemical labeling. J. Neurosci. Methods 173, 27-33 (2008).

96. Pacico, N. \& Mingorance-Le Meur, A. New in vitro phenotypic assay for epilepsy: fluorescent measurement of synchronized neuronal calcium oscillations. PLoS One 9, e84755 (2014).

97. Gurkoff, G. G., Shahlaie, K. \& Lyeth, B. G. In vitro mechanical strain trauma alters neuronal calcium responses: Implications for posttraumatic epilepsy. Epilepsia $\mathbf{5 3}$ Suppl 1, 53-60 (2012).

98. Gala, R., Chapeton, J., Jitesh, J., Bhavsar, C. \& Stepanyants, A. Active learning of neuron morphology for accurate automated tracing of neurites. Front. Neuroanat. 8, 37 (2014).

99. Chen, H., Xiao, H., Liu, T. \& Peng, H. SmartTracing: self-learning-based Neuron reconstruction. Brain Informatics (2015). doi:10.1007/s40708-015-0018-y

100. Chung, K. et al. Structural and molecular interrogation of intact biological systems. Nature 497, 332-7 (2013).

101. Wickersham, I., Lyon, D., Barnard, R. \& Mori, T. Monosynaptic restriction of transsynaptic tracing from single, genetically targeted neurons. Neuron 53, 639-647 (2007).

102. Peng, H. et al. BigNeuron: Large-Scale 3D Neuron Reconstruction from Optical Microscopy Images. Neuron 87, 252-256 (2015).

103. Kay, K. R. et al. Studying synapses in human brain with array tomography and electron microscopy. Nat. Protoc. 8, 1366-80 (2013).

104. Roqué, P. J. et al. in In Vitro Neurotoxicology 758, 361-390 (Springer, 2011).

105. Micheva, K. D. \& Smith, S. J. Array tomography: a new tool for imaging the molecular architecture and ultrastructure of neural circuits. Neuron 55, 25-36 (2007). 
106. Dani, A., Huang, B., Bergan, J., Dulac, C. \& Zhuang, X. Superresolution Imaging of Chemical Synapses in the Brain. Neuron 68, 843-856 (2010).

107. Fan, J., Zhou, X., Dy, J. G., Zhang, Y. \& Wong, S. T. C. An automated pipeline for dendrite spine detection and tracking of 3D optical microscopy neuron images of in vivo mouse models. Neuroinformatics 7, 113-130 (2009).

108. Belinsky, G. S. et al. Patch-clamp recordings and calcium imaging followed by singlecell PCR reveal the developmental profile of 13 genes in iPSC-derived human neurons. Stem Cell Res 12, 101-118 (2014).

109. Hartfield, E. M. et al. Physiological characterisation of human iPS-derived dopaminergic neurons. PLoS One 9, e87388 (2014).

110. Liu, J. et al. Signaling defects in iPSC-derived fragile X premutation neurons. Hum Mol Genet 21, 3795-3805 (2012).

111. Naujock, M. et al. Molecular and functional analyses of motor neurons generated from human cord-blood-derived induced pluripotent stem cells. Stem Cells Dev 23, 30113020 (2014).

112. Nussbaum, J. M., Seward, M. E. \& Bloom, G. S. Alzheimer disease: a tale of two prions. Prion 7, 14-9 (2013).

113. Deisseroth, K. et al. Next-generation optical technologies for illuminating genetically targeted brain circuits. J. Neurosci. 26, 10380-10386 (2006).

114. Godwin, D. W., Che, D., O’Malley, D. M. \& Zhou, Q. Photostimulation with caged neurotransmitters using fiber optic lightguides. J. Neurosci. Methods 73, 91-106 (1997).

115. Grosenick, L., Marshel, J. H. \& Deisseroth, K. Closed-Loop and Activity-Guided Optogenetic Control. Neuron 86, 106-139 (2015).

116. Wilt, B. A. et al. Advances in light microscopy for neuroscience. Annu. Rev. Neurosci. 32, 435-506 (2009).

117. Dombeck, D. A., Harvey, C. D., Tian, L., Looger, L. L. \& Tank, D. W. Functional imaging of hippocampal place cells at cellular resolution during virtual navigation. Nat. Neurosci. 13, 1433-1440 (2010). 